\title{
The Unification of Powerful Quasars and Radio Galaxies and Their Relation to Other Massive Galaxies
}

\section{Citation}

Podigachoski, Pece, Peter Barthel, Martin Haas, Christian Leipski, and Belinda Wilkes. 2015. "The Unification of Powerful Quasars and Radio Galaxies and Their Relation to Other Massive Galaxies." The Astrophysical Journal 806 (1) (June 4): L11. doi:10.1088/2041-8205/806/1/L11.

\section{Published Version}

doi:10.1088/2041-8205/806/1/L11

\section{Permanent link}

http://nrs.harvard.edu/urn-3:HUL.InstRepos:30212127

\section{Terms of Use}

This article was downloaded from Harvard University's DASH repository, and is made available under the terms and conditions applicable to Other Posted Material, as set forth at http:// nrs.harvard.edu/urn-3:HUL.InstRepos:dash.current.terms-of-use\#LAA

\section{Share Your Story}

The Harvard community has made this article openly available.

Please share how this access benefits you. Submit a story.

\section{Accessibility}




\title{
THE UNIFICATION OF POWERFUL QUASARS AND RADIO GALAXIES AND THEIR RELATION TO OTHER MASSIVE GALAXIES
}

\author{
Pece Podigachoski $^{1}$, Peter Barthel ${ }^{1}$, Martin Haas ${ }^{2}$, Christian Leipski $^{3}$, and Belinda Wilkes ${ }^{4}$ \\ ${ }^{1}$ Kapteyn Astronomical Institute, University of Groningen, 9747 AD Groningen, The Netherlands; podigachoski@astro.rug.nl \\ ${ }^{2}$ Astronomisches Institut, Ruhr Universität, D-44801 Bochum, Germany \\ ${ }^{3}$ Max-Planck Institut für Astronomie (MPIA), D-69117 Heidelberg, Germany \\ ${ }^{4}$ Harvard-Smithsonian Center for Astrophysics, Cambridge, MA 02138, USA \\ Received 2015 March 20; accepted 2015 May 12; published 2015 June 4
}

\begin{abstract}
The unification model for powerful radio galaxies (RGs) and radio-loud quasars postulates that these objects are intrinsically the same but viewed along different angles. Herschel Space Observatory data permit the assessment of that model in the far-infrared spectral window. We analyze photometry from Spitzer and Herschel for the distant 3CR hosts, and find that RGs and quasars have different mid-infrared, but indistinguishable far-infrared colors. Both these properties, the former being orientation dependent and the latter orientation invariant, are in line with expectations from the unification model. Adding powerful radio-quiet active galaxies and typical massive starforming $(\mathrm{SF})$ galaxies to the analysis, we demonstrate that infrared colors not only provide an orientation indicator, but can also distinguish active from SF galaxies.
\end{abstract}

Key words: galaxies: active - galaxies: formation - galaxies: high-redshift - galaxies: starburst - infrared: galaxies

\section{INTRODUCTION}

Active galactic nuclei (AGNs) show a large variety of observational properties despite being universally driven by accretion of matter onto supermassive black holes. Their ubiquitous nature and complex interplay with their host galaxies make AGNs essential elements of any model of galaxy evolution. Since their discovery in the 1950s, AGNs have been observationally classified into a number of different types depending on the particular wavelength range used for the observations. Unification models for AGNs (Barthel 1989; Antonucci 1993; Urry \& Padovani 1995) were developed later, explaining the differences between some AGN types solely in terms of viewing angle. An essential part of these models is the so-called AGN "torus": a geometrically and optically thick structure filled with molecular gas and dust surrounding the active nucleus. The AGN torus has a geometry that allows radiation to escape freely in certain directions but not in others, rendering samples of objects selected at X-ray, ultra-violet (UV), and visible wavelengths largely inapplicable for testing unification models.

Radio-selected AGNs are particularly well-suited for testing the unification model, because when selected based on their extended (well outside the torus), low-frequency, transparent (i.e., optically thin) radio emission they show no bias with respect to orientation. The unification model for powerful radio-loud AGNs postulates that radio galaxies (RGs) are viewed edge-on so that the torus obscures the active nucleus and nearby broad-line emitting regions, while quasars (QSRs) are the same objects, but viewed with a faceon torus so that the nuclear regions and broad emission lines are directly visible. The validity of the unification model for powerful radio-loud AGNs has been examined using a variety of orientation-dependent properties (recently reviewed by Antonucci 2012), in the radio (Barthel 1989; Singal 1993), mid-infrared (MIR) (Ogle et al. 2006; Cleary et al. 2007; Haas et al. 2008; Leipski et al. 2010), and X-ray (Wilkes et al. 2013) domains.

\section{INFRARED PROPERTIES}

If the unification model holds, then any isotropic (orientation invariant) property must be comparable for samples of RGs and QSRs with matched low-frequency radio properties. One such isotropic candidate is the far-infrared (FIR) emission, which is dominated by star formation-heated dust on the scale of the host galaxy, but may also include a contribution from optically thin, AGN-powered emission from the torus. Due to sensitivity issues, earlier FIR studies relied on relatively small samples of mostly nearby, less luminous sources, and therefore failed to provide robust conclusions on the unification model for powerful radio-loud AGNs in the FIR (e.g., Hes et al. 1995; van Bemmel et al. 2000; Meisenheimer et al. 2001; Haas et al. 2004). The Herschel Space Observatory ${ }^{5}$ (Pilbratt et al. 2010), with its unprecedented sensitivity, resolution, and wavelength coverage for the first time permits rest-frame FIR tests of unification for distant radio-loud AGNs that have hitherto been outside the reach of infrared space missions. To test the unification model in the FIR, we study the complete $z>13 \mathrm{CR}$ sample of powerful $\left(L_{178 \mathrm{MHz}}>5 \times 10^{28} \mathrm{~W} \mathrm{~Hz}^{-1}\right)$ radio-loud AGNs (Spinrad et al. 1985) using the two Herschel imaging photometers: PACS at 70 and $160 \mu \mathrm{m}$ (Poglitsch et al. 2010) and SPIRE at 250, 350, and $500 \mu \mathrm{m}$ (Griffin et al. 2010). The extreme luminosities of our sample objects ensure that all objects are highly accreting (in quasar-mode, e.g., Best \& Heckman 2012); none of them are classified as a low-ionization emission-line source (e.g., Ogle et al. 2006).

In addition to the Herschel data, the availability of ancillary Spitzer (Werner et al. 2004) data at wavelengths between 3.6 and $24 \mu \mathrm{m}$ (Haas et al. 2008) allows us to obtain physical properties of the 3CR hosts by fitting their full infrared spectral energy distributions (SEDs) with a combination of four components, as explained in detail by Barthel et al. (2012) and Podigachoski et al. (2015). These components include

\footnotetext{
5 Herschel is an ESA space observatory with science instruments provided by European-led Principal Investigator consortia and with important participation from NASA.
} 
Table 1

Properties of the 3CR Objects Plotted in Figure 1

\begin{tabular}{|c|c|c|c|c|c|c|c|}
\hline Name & Type & $z$ & $F_{70 \mu \mathrm{m}} / F_{5 \mu \mathrm{m}}$ & $F_{70 \mu \mathrm{m}} / F_{20 \mu \mathrm{m}}$ & $F_{100 \mu \mathrm{m}} / F_{70 \mu \mathrm{m}}$ & $T_{\text {DUST }}(\mathrm{K})$ & $L_{\mathrm{SF}} / L_{\mathrm{IR}}$ \\
\hline $3 \mathrm{C} 002$ & QSR & 1.04 & 19.2 & 2.3 & 1.3 & 31.6 & 0.23 \\
\hline 3С 014 & QSR & 1.47 & 4.1 & 0.9 & 0.9 & 37.5 & 0.08 \\
\hline $3 \mathrm{C} 068.2$ & $\mathrm{RG}$ & 1.57 & 163.7 & 3.0 & 1.1 & 28.5 & 0.25 \\
\hline 3C 119 & $\mathrm{RG}$ & 1.02 & 15.1 & 1.7 & 0.9 & 37.6 & 0.17 \\
\hline $3 \mathrm{C} 124$ & $\mathrm{RG}$ & 1.08 & 101.4 & 3.4 & 1.1 & 31.9 & 0.30 \\
\hline 3C 190 & QSR & 1.20 & 34.9 & 2.2 & 1.2 & 31.7 & 0.23 \\
\hline $3 \mathrm{C} 205$ & QSR & 1.53 & 11.7 & 1.1 & 0.8 & 40.7 & 0.13 \\
\hline $3 \mathrm{C} 222$ & RG & 1.34 & 1212.7 & 12.6 & 1.3 & 30.6 & 0.62 \\
\hline 3C 245 & QSR & 1.03 & 5.7 & 0.7 & 1.1 & 28.8 & 0.07 \\
\hline 3C 256 & RG & 1.82 & 75.6 & 2.6 & 0.7 & 43.0 & 0.27 \\
\hline $3 \mathrm{C} 257$ & $\mathrm{RG}$ & 2.47 & 38.6 & 4.0 & 1.0 & 38.2 & 0.40 \\
\hline $3 \mathrm{C} 266$ & $\mathrm{RG}$ & 1.27 & 198.3 & 6.8 & 0.8 & 44.2 & 0.60 \\
\hline $3 \mathrm{C} 270.1$ & QSR & 1.52 & 13.0 & 1.4 & 0.7 & 43.5 & 0.15 \\
\hline 3C 297 & RG & 1.41 & 204.5 & 2.9 & 1.5 & 24.9 & 0.30 \\
\hline 3C 298 & QSR & 1.44 & 14.7 & 1.4 & 0.9 & 37.5 & 0.16 \\
\hline $3 C 305.1$ & RG & 1.13 & 43.4 & 3.4 & 0.9 & 34.7 & 0.28 \\
\hline 3C 318 & QSR & 1.57 & 34.0 & 2.9 & 0.9 & 39.6 & 0.31 \\
\hline $3 \mathrm{C} 324$ & RG & 1.21 & 59.3 & 2.2 & 0.7 & 39.6 & 0.23 \\
\hline 3C 368 & RG & 1.13 & 166.8 & 4.0 & 0.9 & 37.3 & 0.38 \\
\hline $3 \mathrm{C} 432$ & QSR & 1.80 & 9.2 & 2.4 & 1.5 & 29.8 & 0.21 \\
\hline 3C 454.0 & QSR & 1.76 & 20.2 & 2.7 & 1.0 & 38.3 & 0.26 \\
\hline 3C 454.1 & RG & 1.84 & 116.4 & 4.7 & 0.9 & 39.3 & 0.45 \\
\hline $3 \mathrm{C} 470$ & $\mathrm{RG}$ & 1.65 & 48.4 & 2.8 & 1.2 & 32.8 & 0.34 \\
\hline \multicolumn{8}{|c|}{ Objects Detected in Only Two Herschel Bands } \\
\hline $3 \mathrm{C} 013$ & RG & 1.35 & 238.8 & 2.0 & $<0.7$ & $\ldots$ & $\ldots$ \\
\hline $3 \mathrm{C} 210$ & RG & 1.17 & 40.7 & 3.0 & $<1.1$ & $\ldots$ & $\cdots$ \\
\hline 3C 220.2 & QSR & 1.16 & 9.8 & 1.0 & $<0.8$ & $\ldots$ & $\ldots$ \\
\hline $3 \mathrm{C} 356$ & RG & 1.08 & 33.7 & 2.0 & $<0.8$ & $\ldots$ & $\cdots$ \\
\hline 3C 469.1 & $\mathrm{RG}$ & 1.34 & 22.9 & 3.0 & $<0.9$ & $\cdots$ & $\cdots$ \\
\hline
\end{tabular}

Note. The top 23 objects are detected in at least three Herschel bands, whereas the bottom five objects in only two Herschel bands. The redshifts of the objects are taken from Spinrad et al. (1985). Columns 4 through 8 are computed by fitting the full infrared spectral energy distributions of the hosts as described in the text.

emission from the circumnuclear, AGN-heated torus dust (Hönig \& Kishimoto 2010), from the extended star formationheated cold dust, from the evolved stellar populations, and from hot nuclear graphite dust. The best-fit SEDs of our 61 3CR hosts were recently presented by Podigachoski et al. (2015). Twenty-three hosts in the sample (presented in Table 1) have good signal-to-noise detections in at least three Herschel bands, typically the three shortest bands, resulting in estimated star formation rates (SFRs) on the order of hundreds of solar masses per year, coeval with the black hole activity (Podigachoski et al. 2015). Such prodigious SFRs, at the level of ultra-luminous infrared galaxies, have similarly been inferred in other studies of high- $z$ RGs (e.g., Ogle et al. 2012; Drouart et al. 2014; Tadhunter et al. 2014).

\section{MIR/FIR COLORS-A POWERFUL TOOL}

A simpler but revealing way to look at the shapes of SEDs is to employ colors. Infrared color-color diagrams are widely used to separate AGNs from non-AGNs in wide-/deep-field galaxy surveys (e.g., Lacy et al. 2004; Stern et al. 2005; Donley et al. 2012; Kirkpatrick et al. 2013). Using the best-fit SEDs of the 23 3CR objects detected in at least three Herschel bands, we create rest-frame color-color diagrams from their hosts' dust emission at 5, 20,70, and $100 \mu \mathrm{m}$. The emission at the former two wavelengths is mainly AGN-powered (e.g., Rowan-Robinson 1995), whereas the emission at the latter two wavelengths is predominantly star formation-powered (Schweitzer et al. 2006).

In Figure 1 we show infrared color-color diagrams for this sample of 13 RGs and 10 QSRs. The distributions of $F_{100 \mu \mathrm{m}} / F_{70 \mu \mathrm{m}}{ }^{6}$ color for RGs and QSRs in Figure 1 are nearly identical, with median values 0.9 . The color coding in Figure 1 (left) demonstrates that the factor of $\sim 2$ scatter around the median FIR colors results from differences in the cold dust temperatures of individual objects, which range from $\sim 25$ to $\sim 45 \mathrm{~K}$ (Podigachoski et al. 2015). The emission from hosts with dust temperatures $<33 \mathrm{~K}$ peaks at wavelengths $>70 \mu \mathrm{m}$ resulting in $F_{100 \mu \mathrm{m}} / F_{70 \mu \mathrm{m}}>1$. We apply both maximum likelihood and classical methods to statistically compare the measured RG and QSR $F_{100 \mu \mathrm{m}} / F_{70 \mu \mathrm{m}}$ colors. Assuming that the colors are normally distributed, we compute the parameters of the distributions that maximize the probability of measuring such colors. The means and standard deviations of these maximum likelihood distributions are 1.0 and 0.2, respectively, for both the RGs and QSRs. The corresponding maximum likelihood cumulative distribution functions and the empirical (measured) cumulative distributions are presented in Figure 2 (left). The Kolmogorov-Smirnov (KS) test reveals that the two samples are drawn from the same distribution $\left(\mathrm{KS}_{\text {statistic }}=0.07, p=1\right)$. We conclude that the FIR colors of these 3CR RGs and QSRs are indistinguishable (i.e.,

\footnotetext{
6 Monochromatic flux densities are given in units of $\mathrm{erg} \mathrm{s}^{-1} \mathrm{~cm}^{-2} \mathrm{~Hz}^{-1}$.
} 

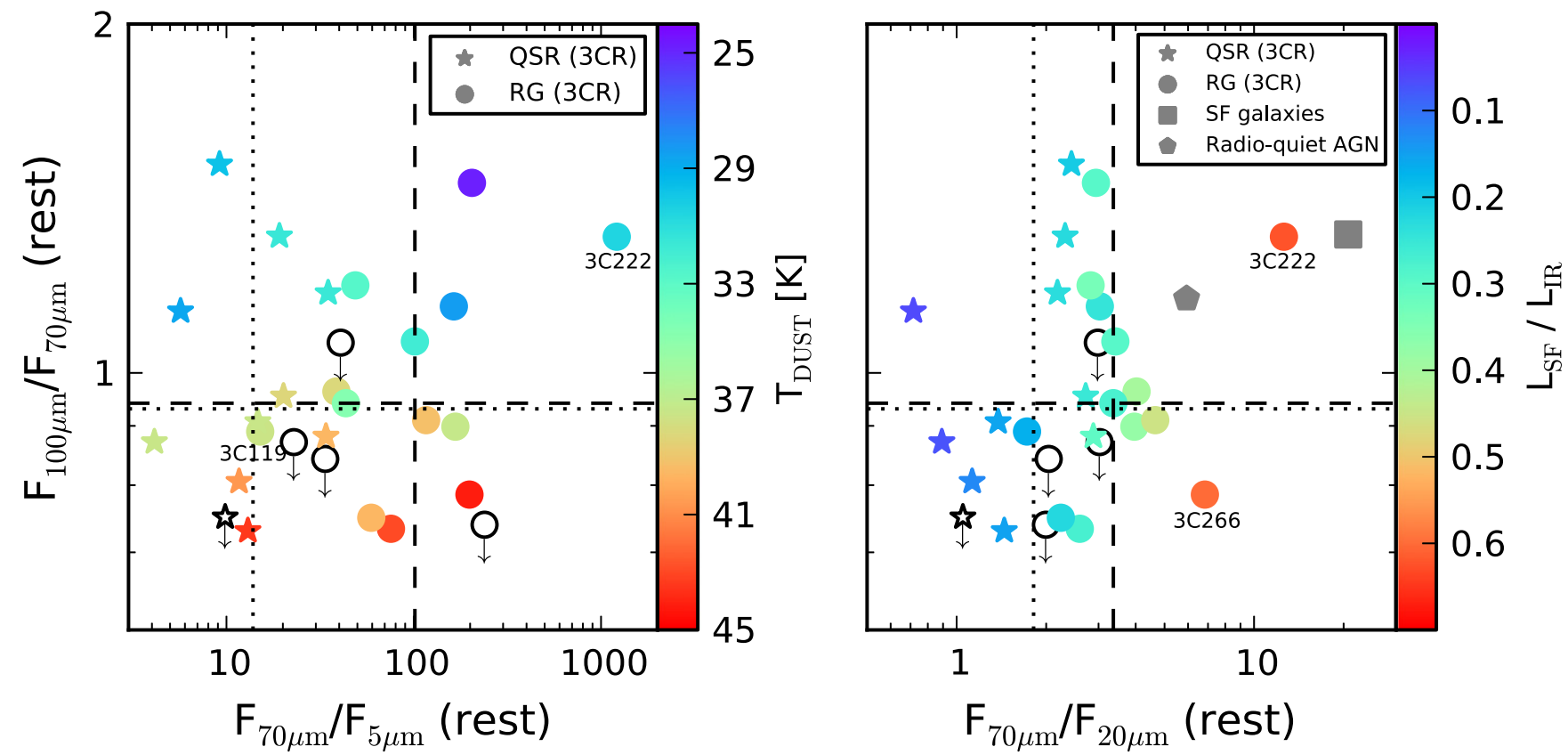

Figure 1. Rest-frame infrared color-color diagrams computed by fitting a multi-component model to the observed spectral energy distributions of the $3 \mathrm{CR}$ objects. The ordinate represents $F_{100 \mu \mathrm{m}} / F_{70 \mu \mathrm{m}}$ color in both plots, the abscissa the $F_{70 \mu \mathrm{m}} / F_{5 \mu \mathrm{m}}$ (left plot) and $F_{70 \mu \mathrm{m}} / F_{20 \mu \mathrm{m}}$ color (right plot). The 3CR quasars are shown with stars, the 3CR radio galaxies with circles. Filled colored symbols represent objects detected in at least three Herschel bands, whereas empty symbols represent objects detected in only the two shortest Herschel bands, for which $F_{100 \mu \mathrm{m}}$ is estimated as an upper limit. Dotted and dashed lines mark the corresponding median colors of quasars and radio galaxies, respectively. (Left) the $3 \mathrm{CR}$ objects are color-coded according to their cold dust temperatures, $T_{\mathrm{DusT}}$, as found from the SED-fitting. The TDUST scale is indicated in the left vertical color bar. (Right) the $3 \mathrm{CR}$ objects are color-coded according to their fractional star formation luminosity, $L_{\mathrm{SF}} / L_{\mathrm{IR}}$. $T_{\mathrm{DUST}}$ and $L_{\mathrm{SF}} / L_{\mathrm{IR}}$ are listed in Table 1. The LSF/LIR scale is indicated in the right vertical color bar. Gray symbols show the median colors of the comparison star-forming galaxies at $z \sim 2$ (square) and radio-quiet AGN (pentagon), both from Kirkpatrick et al. (2012).

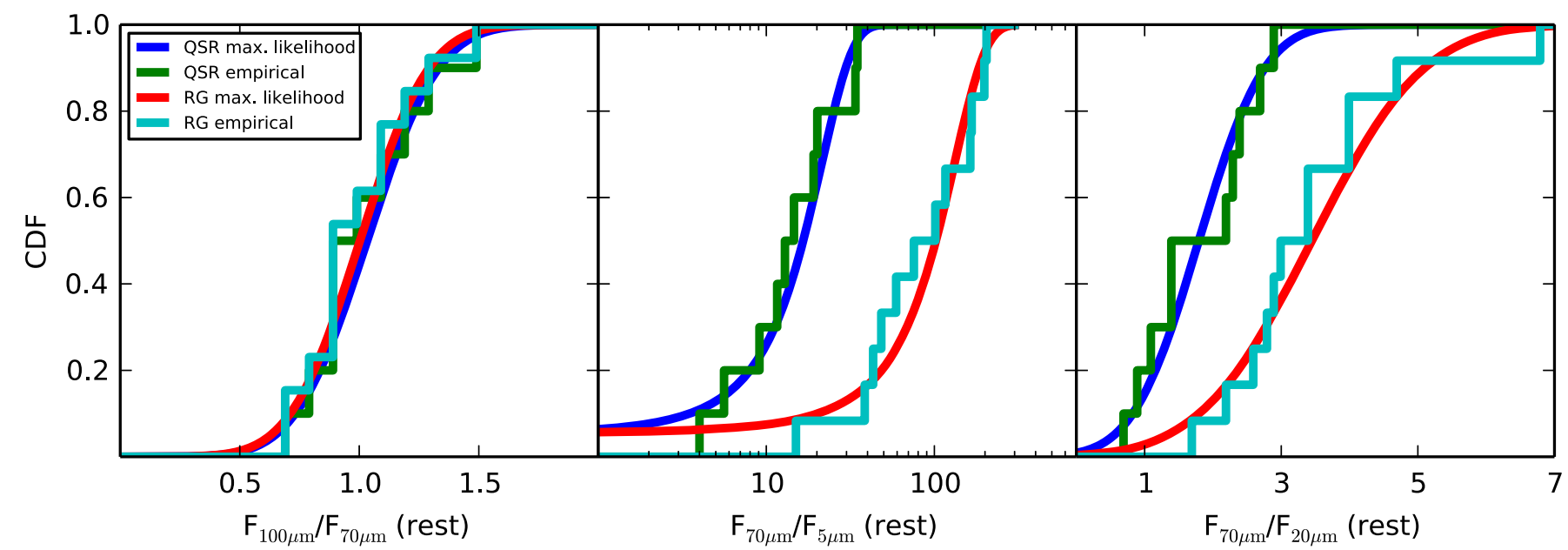

Figure 2. Maximum likelihood and empirical (measured) cumulative distribution functions of $F_{100 \mu \mathrm{m}} / F_{70 \mu \mathrm{m}}\left(\right.$ left), $F_{70 \mu \mathrm{m}} / F_{5 \mu \mathrm{m}}(\mathrm{middle})$, and $F_{70 \mu \mathrm{m}} / F_{20 \mu \mathrm{m}}($ right $)$ colors of the 3CR objects. Quasars (blue/green) and radio galaxies (red/cyan) have different $F_{70 \mu \mathrm{m}} / F_{5 \mu \mathrm{m}}$ and $F_{70 \mu \mathrm{m}} / F_{20 \mu \mathrm{m}}$ distributions and indistinguishable $F_{100 \mu \mathrm{m}} / F_{70 \mu \mathrm{m}}$ distributions, both in line with expectations from the unification model of radio-loud AGN. (Left) parameters of quasar maximum likelihood distribution: $\mu=1.0, \sigma=0.2$. Parameters of radio galaxy maximum likelihood distribution: $\mu=1.0, \sigma=0.2$. (Middle) parameters of quasar maximum likelihood distribution: $\mu=16.6, \sigma=10.2$. Parameters of radio galaxy maximum likelihood distribution: $\mu=102.1, \sigma=63.9$. (Right) parameters of quasar maximum likelihood distribution: $\mu=1.8, \sigma=0.7$. Parameters of radio galaxy maximum likelihood distribution: $\mu=3.4, \sigma=1.3$. 3C 222 has not been included in the $F_{70 \mu \mathrm{m}} / F_{5} \mu \mathrm{m}$ and $F_{70 \mu \mathrm{m}} / F_{20 \mu \mathrm{m}}$ distributions of radio galaxies (see text).

orientation invariant), as expected in the unification model for AGN host galaxies with similar SFRs.

In contrast, the RGs and QSRs show clear separation in their $F_{70 \mu \mathrm{m}} / F_{5 \mu \mathrm{m}}$ colors (Figure 1, left): their median colors are 101.4 and 13.9, respectively. One RG, 3C 119, has colors that overlap the QSRs in Figure 1 (left), not surprisingly, because 3C 119 is a well-known (de Vries et al. 1997) borderline source where some of the hot dust is visible, and the UV/visible AGN continuum is only partly hidden. The $5 \mu \mathrm{m}$ emission of another RG, 3C 222, is only weakly constrained (Podigachoski et al. 2015), resulting in an unusually red $F_{70 \mu \mathrm{m}} / F_{5 \mu \mathrm{m}}$ color for this object. Considering this particular value as an upper limit, we obtain $\mathrm{KS}_{\text {statistic }}=0.92\left(p=5 \times 10^{-5}\right)$, and maximum likelihood distributions as plotted in Figure 2 (middle). These 
clearly different distributions are driven by the differences between the RG and QSR SEDs at $5 \mu \mathrm{m}$. RGs are fainter than QSRs at $5 \mu \mathrm{m}$, as expected if their hot inner torus dust emission is obscured due to its edge-on viewing angle (Haas et al. 2008; Leipski et al. 2010; Dicken et al. 2014). Our results support the hypothesis that $F_{70 \mu \mathrm{m}} / F_{5 \mu \mathrm{m}}$ to a large extent reveals the level of obscuration in powerful radio-loud objects, and as such is an orientation indicator for that AGN population. However, intrinsic differences in this ratio for individual objects are not ruled out, and may contribute to the observed scatter.

Although our adopted SED-fitting approach is physically grounded and provides us with a close approximation of the objects' infrared SEDs, we further explore the robustness of the results presented above without using dust emission models. To this end, we employ a simple (model-independent) linear interpolation of the observed flux values to obtain the restframe colors and create the color-color diagrams. We apply this method to the sample of objects detected in at least three Herschel bands, and find that the RGs and QSRs remain clearly separated in the $F_{70 \mu \mathrm{m}} / F_{5 \mu \mathrm{m}}$ color, with median values of 83.9 and 12.3 , respectively. Their respective median $F_{100 \mu \mathrm{m}} / F_{70 \mu \mathrm{m}}$ colors (1.0 and 0.9) remain indistinguishable, similarly to the results obtained when following the SED-fitting approach.

In addition to the $233 \mathrm{CR}$ hosts detected in at least three Herschel bands, seven hosts are detected in only the two PACS bands while remaining below the detection limit in the SPIRE bands. Given the low redshift range $(1<z \leqslant 1.35)$ of five of these seven hosts, the PACS $160 \mu \mathrm{m}$ band probes their cold dust emission at rest-frame $\sim 70 \mu \mathrm{m}$ allowing their inclusion (empty symbols) in the color-color diagrams presented in Figure 1. While this inclusion does not change the trends discussed above, their $F_{70 \mu \mathrm{m}} / F_{5 \mu \mathrm{m}}$ colors tend to be somewhat bluer than the median colors of the 23 objects detected in at least three Herschel bands. This is likely a consequence of their lower levels of star formation (Podigachoski et al. 2015). As such, it is important to note that the present FIR test of unification can directly be applied only to the long wavelength detected, i.e., the star-forming (SF) objects from the high-z 3CR sample. The 31 remaining hosts from the sample that are either detected only in the PACS $70 \mu \mathrm{m}$ band or not detected at all, have weak or absent star formation and thus no definitive conclusions can be drawn. Nevertheless, the non-detection fractions at SPIRE $250 \mu \mathrm{m}$ for RGs and QSRs are comparable ( $\sim 65 \%$ and $\sim 58 \%$, respectively), and thus do not represent evidence against the unification model.

The high level of obscuration in the MIR prevents the $F_{70 \mu \mathrm{m}} / F_{5 \mu \mathrm{m}}$ color from directly probing the relative importance of the SF and AGN activity. We therefore use the AGNpowered circumnuclear dust emission at $20 \mu \mathrm{m}$ (near the peak emission from the torus, e.g., Hönig \& Kishimoto 2010), and employ the $F_{70 \mu \mathrm{m}} / F_{20 \mu \mathrm{m}}$ color to probe the dominant power source in the infrared. As shown in Figure 1 (right), the $F_{70 \mu \mathrm{m}}$ $/ F_{20 \mu \mathrm{m}} \mathrm{RG}$ and QSR colors are closer to each other than the $F_{70 \mu \mathrm{m}} / F_{5 \mu \mathrm{m}}$ colors, but still have an offset, with median values of 3.4 and 1.8 , respectively $\left(\mathrm{KS}_{\text {statistic }}=0.65, p=0.01\right.$; see Figure 2, right), confirming that the level of obscuration at $20 \mu \mathrm{m}$ is substantially lower than that at $5 \mu \mathrm{m}$. The symbols in Figure 1 (right) are color-coded according to the fractional star formation luminosity, $L_{\mathrm{SF}} / L_{\mathrm{IR}}$, as inferred by Podigachoski et al. (2015). This color-coding demonstrates that, despite the strong star formation activity of several hundreds of solar masses per year, the total infrared luminosity of the 3CR hosts is generally dominated by their AGN activity, regardless if that activity is obscured or not (Podigachoski et al. 2015). The only exceptions are two RGs, 3C 266 and 3C 222, which have similar $L_{\mathrm{SF}}$ but lower IR AGN luminosities than the other RGs (Podigachoski et al. 2015). However, a general increase in the $L_{\mathrm{SF}} / L_{\mathrm{IR}}$ ratio of $3 \mathrm{CR}$ objects, from $\sim 10 \%$ to $\sim 60 \%$, is observed when progressing to larger $F_{70 \mu \mathrm{m}} / F_{20 \mu \mathrm{m}}$ values, i.e., to redder colors.

We examine this trend further by comparing the 3CR AGN infrared colors to those of typical SF galaxies where the infrared emission draws uniquely from star formation. The SFR strongly correlates with stellar mass and redshift (Elbaz et al. 2007); we therefore select $z \sim 2$ SF galaxies from Kirkpatrick et al. (2012) with stellar masses and redshifts comparable to those of the 3CR hosts (which have $M_{\text {stellar }} \gtrsim 10^{11} M_{\odot}$, e.g., Best et al. 1998; de Breuck et al. 2010). Given that stars in these SF galaxies are formed at rates (Kirkpatrick et al. 2012) comparable to those in the $3 \mathrm{CR}$ hosts, the $L_{\mathrm{SF}} / L_{\mathrm{IR}} \sim 1$ of the SF galaxies are consistent with their red $F_{70 \mu \mathrm{m}} / F_{20 \mu \mathrm{m}}$ colors (Figure 1 right). The bluer $F_{70 \mu \mathrm{m}} / F_{20 \mu \mathrm{m}}$ colors of the powerful 3CR AGNs are likely a consequence of the strong torus emission at $20 \mu \mathrm{m}$. The average cold dust temperature of the SF galaxies is $28 \pm 2 \mathrm{~K}$ (Kirkpatrick et al. 2012), hence their FIR colors are also consistent with the temperature trends found for the $3 \mathrm{CR}$ hosts. The $F_{70 \mu \mathrm{m}} / F_{20 \mu \mathrm{m}}$ colors of powerful AGNs are offset from those of SF galaxies, and hence, in addition to being indicators of orientation, FIR/MIR colors also distinguish between active and SF galaxies in the most luminous SF objects, analogous to the case for local Seyfert galaxies (e.g., de Grijp et al. 1985).

A limitation for the general unification discussion is the fact that radio-loud AGNs, such as the 3CR sources investigated here, represent a small $(\sim 10 \%)$ fraction of the complete AGN population. It is therefore important to compare the colors of the powerful $3 \mathrm{CR}$ radio-loud AGNs to those of powerful radio-quiet AGNs. To achieve this, we select a sample of distant $\left(z_{\text {med }} \sim 1.9\right)$, luminous, radio-quiet AGNs $\left(L_{2-10 \mathrm{keV}} \sim 5 \times 10^{44} \mathrm{erg} \mathrm{s}^{-1}\right)$ hosted by massive $\left(M_{\text {stellar }} \sim 10^{11} M_{\odot}\right)$ SF galaxies, from Kirkpatrick et al. (2012). As shown in Figure 1 (right) the radio-quiet AGNs have $F_{70 \mu \mathrm{m}} / F_{20 \mu \mathrm{m}}$ colors redder than those of the 3CR RGs, but bluer than those of the SF galaxies. Given that the SF activity in these radio-quiet AGNs accounts for $56 \%$ of the total infrared luminosity (Kirkpatrick et al. 2012), this finding agrees with the trend of increasing $L_{\mathrm{SF}} / L_{\mathrm{IR}}$ toward larger $F_{70 \mu \mathrm{m}} / F_{20 \mu \mathrm{m}}$ values for the $3 \mathrm{CR}$ objects discussed above.

\section{CONCLUSION}

In conclusion, far- to mid-infrared colors represent a useful diagnostic of the orientation of powerful radio-loud AGNs, and support the unification model for these objects. At wavelengths longer than $20 \mu \mathrm{m}$, where obscuration is a small effect, these colors are also indicators of the relative contributions of star formation and nuclear activity in galaxies. These diagnostics may prove useful in future studies of large samples of dusty SF galaxies/AGNs to investigate the duty cycle of nuclear activity, feedback, and AGN and galaxy growth. 
We thank the referee, Patrick Ogle, for a very constructive report which improved the manuscript. Data were taken from the Herschel Guaranteed Time project The Herschel Legacy of distant radio-loud AGN (PI: PB). P.P. acknowledges the Nederlandse Organisatie voor Wetenschappelijk Onderzoek (NWO) for a PhD fellowship. The Herschel spacecraft was designed, built, tested, and launched under a contract to ESA managed by the Herschel/Planck Project team by an industrial consortium under the overall responsibility of the prime contractor Thales Alenia Space (Cannes), and including Astrium (Friedrichshafen) responsible for the payload module and for system testing at spacecraft level, Thales Alenia Space (Turin) responsible for the service module, and Astrium (Toulouse) responsible for the telescope, with in excess of a hundred subcontractors. PACS has been developed by a consortium of institutes led by MPE (Germany) and including UVIE (Austria); KU Leuven, CSL, IMEC (Belgium); CEA, LAM (France); MPIA (Germany); INAF-IFSI/OAA/OAP/OAT, LENS, SISSA (Italy); IAC (Spain). This development has been supported by the funding agencies BMVIT (Austria), ESA-PRODEX (Belgium), CEA/CNES (France), DLR (Germany), ASI/INAF (Italy), and CICYT/MCYT (Spain). SPIRE has been developed by a consortium of institutes led by Cardiff University (UK) and including Univ. Lethbridge (Canada); NAOC (China); CEA, LAM (France); IFSI, Univ. Padua (Italy); IAC (Spain); Stockholm Observatory (Sweden); Imperial College London, RAL, UCL-MSSL, UKATC, Univ. Sussex (UK); and Caltech, JPL, NHSC, Univ. Colorado (USA). This development has been supported by national funding agencies: CSA (Canada); NAOC (China); CEA, CNES, CNRS (France); ASI (Italy); MCINN (Spain); SNSB (Sweden); STFC, UKSA (UK); and NASA (USA). This work is partly based on observations made with the Spitzer Space Telescope, which is operated by the Jet Propulsion Laboratory, California Institute of Technology under a contract with NASA.

Facilities: Herschel, Spitzer.

\section{REFERENCES}

Antonucci, R. 1993, ARA\&A, 31, 473

Antonucci, R. 2012, A\&AT, 27, 557

Barthel, P. D. 1989, ApJ, 336, 606

Barthel, P., Haas, M., Leipski, C., \& Wilkes, B. 2012, ApJL, 757, LL26

Best, P. N., \& Heckman, T. M. 2012, MNRAS, 421, 1569

Best, P. N., Longair, M. S., \& Röettgering, H. J. A. 1998, MNRAS, 295, 549

Cleary, K., Lawrence, C. R., Marshall, J. A., Hao, L., \& Meier, D. 2007, ApJ, 660,117

de Breuck, C., Seymour, N., Stern, D., et al. 2010, ApJ, 725, 36

de Grijp, M. H. K., Miley, G. K., Lub, J., \& de Jong, T. 1985, Natur, 314,240

de Vries, W. H., O’Dea, C. P., Baum, S. A., et al. 1997, ApJS, 110, 191

Dicken, D., Tadhunter, C., Morganti, R., et al. 2014, ApJ, 788, 98

Donley, J. L., Koekemoer, A. M., Brusa, M., et al. 2012, ApJ, 748, 142

Drouart, G., de Breuck, C., Vernet, J., et al. 2014, A\&A, 566, AA53

Elbaz, D., Daddi, E., le Borgne, D., et al. 2007, A\&A, 468, 33

Griffin, M. J., Abergel, A., Abreu, A., et al. 2010, A\&A, 518, LL3

Haas, M., Müller, S. A. H., Bertoldi, F., et al. 2004, A\&A, 424, 531

Haas, M., Willner, S. P., Heymann, F., et al. 2008, ApJ, 688, 122

Hes, R., Barthel, P. D., \& Hoekstra, H. 1995, A\&A, 303, 8

Hönig, S. F., \& Kishimoto, M. 2010, A\&A, 523, AA27

Kirkpatrick, A., Pope, A., Alexander, D. M., et al. 2012, ApJ, 759, 139

Kirkpatrick, A., Pope, A., Charmandaris, V., et al. 2013, ApJ, 763, 123

Lacy, M., Storrie-Lombardi, L. J., Sajina, A., et al. 2004, ApJS, 154, 166

Leipski, C., Haas, M., Willner, S. P., et al. 2010, ApJ, 717, 766

Meisenheimer, K., Haas, M., Müller, S. A. H., et al. 2001, A\&A, 372, 719

Ogle, P., Davies, J. E., Appleton, P. N., et al. 2012, ApJ, 751, 13

Ogle, P., Whysong, D., \& Antonucci, R. 2006, ApJ, 647, 161

Pilbratt, G. L., Riedinger, J. R., Passvogel, T., et al. 2010, A\&A, 518, LL1

Podigachoski, P., Barthel, P. D., Haas, M., et al. 2015, A\&A, 575, AA80

Poglitsch, A., Waelkens, C., Geis, N., et al. 2010, A\&A, 518, LL2

Rowan-Robinson, M. 1995, MNRAS, 272, 737

Schweitzer, M., Lutz, D., Sturm, E., et al. 2006, ApJ, 649, 79

Singal, A. K. 1993, MNRAS, 262, L27

Spinrad, H., Marr, J., Aguilar, L., \& Djorgovski, S. 1985, PASP, 97, 932

Stern, D., Eisenhardt, P., Gorjian, V., et al. 2005, ApJ, 631, 163

Tadhunter, C., Dicken, D., Morganti, R., et al. 2014, MNRAS, 445, L51

Urry, C. M., \& Padovani, P. 1995, PASP, 107, 803

van Bemmel, I. M., Barthel, P. D., \& de Graauw, T. 2000, A\&A, 359, 523

Werner, M. W., Roellig, T. L., Low, F. J., et al. 2004, ApJS, 154, 1

Wilkes, B. J., Kuraszkiewicz, J., Haas, M., et al. 2013, ApJ, 773, 15 